\title{
The clinical utility of circulating neuroendocrine gene transcript analysis in well-differentiated paragangliomas and pheochromocytomas
}

\author{
M Pęczkowska', J Cwikla², M Kidd³ , A Lewczuk4, A Kolasinska-Ćwikła5', D Niec', \\ I Michałowska', A Prejbisz', A Januszewicz', J Chiarelli' ${ }^{3}$, L Bodei ${ }^{6}$ and I Modlin ${ }^{7}$ \\ ${ }^{1}$ Institute of Cardiology, Warsaw, Poland, ${ }^{2}$ University of Warmia and Mazury, The Faculty of Medical \\ Sciences, Olsztyn, Poland, ${ }^{3}$ Wren Laboratories, Branford, Connecticut, USA, ${ }^{4}$ Medical University of \\ Gdansk, Gdansk, Poland, ${ }^{5}$ Maria Skłodowska-Curie Memorial Cancer Center, Warsaw, Poland, \\ ${ }^{6}$ Memorial Sloan Kettering Cancer Center, New York, USA, and ${ }^{7}$ Yale University School of Medicine, \\ New Haven, Connecticut, USA
}

\begin{abstract}
Context: Paragangliomas and pheochromocytomas (PPGLs) exhibit variable malignancy, which is difficult to determine by histopathology, amine measurements or tissue genetic analyses.

Objective: To evaluate whether a 51-neuroendocrine gene blood analysis has clinical utility as a diagnostic and prognostic marker.

Design: Prospective cohort study. Well-differentiated PPGLs $(n=32)$, metastatic $(n=4) ; \operatorname{SDH} x$ mutation $(n=25) ; 12$ biochemically active, Lanreotide treated $(n=4)$. Nine patients had multiple sampling. Age- and gender-matched controls and GEP-NETs (comparators).

Methods: Circulating neuroendocrine tumor mRNA measured (qPCR) with multianalyte algorithmic analysis. Metabolic, epigenomic and proliferative genes as well as somatostatin receptor expression were assessed (averaged, normalized gene expression: mean \pm S.E.M.). Amines were measured by HPLC and chromogranin A by ELISA. Analyses (2-tailed): Fisher's test, non-parametric (Mann-Whitney), receiver-operator curve (ROC) and multivariate analysis (MVA). All data are presented as mean \pm S.E.M.

Results: PPGL were NETest positive (100\%). All exhibited higher scores than controls $(55 \pm 5 \%$ vs $8 \pm 1 \%, P=0.0001)$, similar to GEP-NETs $(47 \pm 5 \%)$. ROC analysis area under curve was 0.98 for differentiating PPGLs/controls (cut-off for normal: $26.7 \%)$. Mutation status was not directly linked to NETest. Genetic and molecular clustering was associated $(P<0.04)$ with NETest scores. Metastatic $(80 \pm 9 \%)$ and multicentric $(64 \pm 9 \%)$ disease had significantly $(P<0.04)$ higher scores than localized disease (43 $\pm 7 \%)$. Progressive disease (PD) had the highest scores $(86 \pm 2 \%)$ vs stable (SD, $41 \pm 2 \%)$ $(P<0.0001)$. The area under the curve for PD from SD was 0.93 (cut-off for PD: 53\%). Proliferation, epigenetic and somatostatin receptor gene expression was elevated $(P<0.03)$ in PD. Metabolic gene expression was decreased in SDHx mutations. Repeat NETest measurements defined clinical status in the 9 patients (6 SD and 3 PD). Amine measurement was non-informative. Multivariate analysis identified NETest $>53 \%$ as an independent prognostic factor. Conclusion: Circulating NET transcript analysis is positive (100\% diagnostic) in well-differentiated PCC/PGL, scores were elevated in progressive disease irrespective of mutation or biochemical activity and elevated levels were prognostic.
\end{abstract}

() 2017 European Society of Endocrinology Printed in Great Britain 


\section{Introduction}

Paragangliomas (PGLs) and pheochromocytomas (PCCs) are complex neuroendocrine lesions that are ubiquitous with an incidence of 2/1000000 (1). They exhibit variable malignancy, which is not always easy to delineate by histopathology. The key clinical unmet needs include accurate determination of malignancy, identification of residual disease and the introduction of tools to accurately monitor disease progression and evaluate treatment response.

Paragangliomas and pheochromocytomas (commonly called PPGLs) arise from chromaffin cells (adrenal medulla: PCCs) or histologically similar cells in the parasympathetic and sympathetic ganglia (PGLs). The abdomino-thoracic tumors often secrete amines or their metabolites $(2,3)$ and are associated with hypertension and symptoms including palpitations, sweating, flushing, fatigue, nausea, irregular bowel function and cardiac involvement (3). Genetically, PPGLs are categorized into PGL1-5 based on mutations in $S D H x /$ hypoxia pathway or in $V H L, R E T 1$ and $N F-1$, pathways respectively $(4,5)$. Approximately $40 \%$ of PPGLs are due to germline mutations (4), whereas mutations in $S D H B$ are the most common genetic association with metastasis (6). The majority of PPGLs (70\%) are well differentiated but 10-30\% metastasize (7).

Despite being well characterized genetically, susceptibility genes for PPGL malignancy remain unknown. In addition, standard histopathological approaches, e.g., the Ki67 index, have not proven to be reliable as a predictor of tumor growth or metastasis (7). One consequence of this is the need of a scientific tool to identify high-risk and/or poor prognosis tumors. Two scoring systems have been developed, the Pheochromocytoma of the Adrenal Scaled Score (PASS) (8) and the Grading of Adrenal Pheochromocytoma and Paraganglioma (GAPP) criteria (7). The former was only developed for PCCs and was poorly concordant between pathologists in a validation study (9). The latter has not been validated, includes biochemical measures, which are of variable utility (10), and its development did not include tumor genetic information including $S D H B$ mutational status (11).

This manuscript assesses an alternative to tissue-based predictive tools using multianalyte algorithmic analysis of blood-based tumor transcripts. The most common current diagnostic strategy for PPGLs is the measurement of urine and plasma metanephrine and nor-metanephrine levels $(12,13)$. However, poor metrics due to incorrect collection procedures can obfuscate utility (10). In addition, many tumors do not exhibit increased values of these analytes, and little data support the use of these measurements either for residual disease monitoring or treatment efficacy assessment (14). Other blood-based biomarkers, e.g., plasma miRNA or circulating free mutant DNA, have not been evaluated. Accurate methods for early identification of disease recurrence or tumor progression remain a key clinical issue.

Recently, it has become apparent to the oncological community that cell secretory products alone are inadequate descriptors of neoplastic cell biology. This has led to the development of multiple analyte measurements to better define the diversity of the neoplastic environment. Such assays, integrated with mathematical algorithmic analyses (MAAA) more accurately capture the magnitude and multiplicity of biological information inherent in a neoplasm (15). MAAA therefore provides the basis for biomarker development that can provide insight into tumor characteristics, level of malignancy and likelihood of metastasis (16). This strategy encompasses the hypothesis that neoplastic behavior is embodied in the 'Hallmarks of Cancer' descriptor provided by Hanahan and Weinberg. These characteristics include proliferation, evading apoptosis, pluripotency, metabolism and secretion (17).

A blood-based MAAA (NETest) has recently been developed for neuroendocrine tumors (NETs) $(18,19,20)$ and demonstrated to have utility in identifying residual disease (21), defining progression (22) and predicting treatment efficacy (23). NETs share a number of similarities with PPGLs. They arise from enteroendocrine cells, secrete amines, are associated with similar symptoms (flushing and diarrhea) and exhibit an overlap in mutational landscape e.g., SDHB, VHL and NF-1 $(24,25)$, as well as exhibiting common downstream transcriptional and pathway abnormalities (e.g., hypoxia and growth factor Ras/Raf signaling) (26). In GEP-NETs, gene expression defines different cut-offs e.g., minimal activity: <0-14\%, low activity: $14-40 \%$ and intermediate-high activity: $>40-100 \%$ (18). In addition, clinically progressive disease in GEP-NETs is identified by elevated expression of a number of oncologically relevant gene clusters including those involved in proliferation, metabolism, the epigenome, some secretory genes, somatostatin receptor expression and pluripotency (18).

Based on the recognition of this degree of commonality, we sought to evaluate whether neuroendocrine tumor transcript (NETest) levels in blood could identify well- 
differentiated PPGLs i.e., were detectable (diagnostic) and whether this information had clinical relevance (identification of disease progression). In addition, as the clinically relevant cut-offs (for the NETest) as well as the genes involved in defining malignancy are not known in PPGLs, we, also, evaluated these parameters.

\section{Subjects and methods}

\section{Approach}

To assess the value of the circulating mRNA signature in PPGLs, we prospectively collected blood samples from 32 patients with known mutation status, mostly with the Polish founding mutation (SDHD: c.33 C/A C11X) (27) at two centers (Poland/Warsaw and Gdansk) over a 15-month period. These samples were used to evaluate the NETest as a 'diagnostic' and identify whether measurements correlated with clinical disease or mutation status. More than one blood sample was available from a subset of patients $(n=9)$. These were correlated with outcome to evaluate the clinical utility of the NETest.

The NETest captures gene expression of cancer hallmarks including proliferation and metabolism, which are clinically relevant to disease activity and progression (17). We therefore specifically focused on these in a subset analysis. We compared the NETest to standard catecholamine measurements (free normetanephrine, metanephrine and methoxytyramine in plasma) as well as to $\mathrm{CgA}$ as measurement of this neuroendocrine secretory marker is considered clinically useful in PPGLs (28). Tumors secreting methoxytyramine have been classified as a dopaminergic, secreting normetanephrine as noradrenergic and metanephrine as adrenergic.

All patients provided informed consent for the blood translational analysis authorized by the local ethics committee at both institutions. The NETest results from the PPGL cohort were compared (1:1) to genderand age- (to within 5 years) matched NETs and controls $(n=32$ each $)$.

\section{Patients}

Histopathologically confirmed PPGLs $(n=32)$ (collected between May 2015 and July 2016) were studied. Patient demographics are summarized in Table 1 . All tumors were well differentiated. The Ki-67 index was low in all tumors in which it was measured ( $n=13$ : median: $2 \%$, range: $1-2 \%$ except one (SDHB mutation) where the Ki67 was $16.3 \%$ ). Four (13\%) exhibited distant metastases. The mean age
Table 1 Demographics of the PPGL $(n=32)$, matched GEP and BP NET comparator $(n=32)$ cohorts. Data are presented as $n(\%)$.

\begin{tabular}{|c|c|}
\hline Demographics & Values \\
\hline \multicolumn{2}{|l|}{ PPGL cohort } \\
\hline Age (years) (mean/range) & $34(12-62)$ \\
\hline Gender (M:F) & $17: 15$ \\
\hline \multicolumn{2}{|l|}{ Tumor type } \\
\hline PCC & $4(13 \%)$ \\
\hline Mixed PCC/PGL & $10(31 \%)$ \\
\hline PGL & $18(56 \%)$ \\
\hline Functional (amine, metabolite secretion) & $15(47 \%)$ \\
\hline Dopamine & $7(22 \%)^{*}$ \\
\hline Noradrenaline & $9(28 \%) * *$ \\
\hline Chromogranin A & $8(25 \%)$ \\
\hline Normetanephrine & $4(13 \%)$ \\
\hline Metanephrine & $2(6 \%)$ \\
\hline Methoxytyramine & $4(13 \%)$ \\
\hline \multicolumn{2}{|l|}{ Disease extent } \\
\hline Local & $18(56 \%)$ \\
\hline Multicentric & $7(22 \%)$ \\
\hline Metastatic & $4(12 \%)$ \\
\hline \multicolumn{2}{|l|}{ Disease status (RECIST 1.0) } \\
\hline NED & $2(6 \%)$ \\
\hline SD & $19(59 \%)$ \\
\hline PD & $11(35 \%)$ \\
\hline \multicolumn{2}{|l|}{ Therapy } \\
\hline Surgery & $25(78 \%)$ \\
\hline Embolization & $5(16 \%)$ \\
\hline PRRT & $5(16 \%)$ \\
\hline EBRT & $3(9 \%)$ \\
\hline Brachytherapy & $1(3 \%)$ \\
\hline SSA & $4(12 \%)^{\#}$ \\
\hline Currently no treated & $28(88 \%)^{\#}$ \\
\hline \multicolumn{2}{|l|}{ Matched GEP and BP NET comparator cohort } \\
\hline Age (years) (mean/range) & $38(27-61)$ \\
\hline Gender (M:F) & $17: 15$ \\
\hline \multicolumn{2}{|l|}{ Tumor type } \\
\hline Small intestine & $19(59 \%)$ \\
\hline Pancreas & $4(13 \%)$ \\
\hline Broncho-pulmonary & $3(9 \%)$ \\
\hline Stomach & $1(3 \%)$ \\
\hline Duodenum & $2(6 \%)$ \\
\hline Appendix & $1(3 \%)$ \\
\hline Rectum & $1(3 \%)$ \\
\hline MEN-1 & $1(3 \%)$ \\
\hline \multicolumn{2}{|l|}{ Grade } \\
\hline GEP & $20(63 \%)$ \\
\hline \multicolumn{2}{|l|}{ G1 } \\
\hline $\mathrm{G} 2$ & $8(25 \%)$ \\
\hline G3 & $1(3 \%)$ \\
\hline \multicolumn{2}{|l|}{$\mathrm{BP}$} \\
\hline Typical carcinoid & $2(6 \%)$ \\
\hline Atypical carcinoid & $1(3 \%)$ \\
\hline LCNEC/SCLC & $0(0 \%)$ \\
\hline \multicolumn{2}{|l|}{ Disease extent } \\
\hline Metastatic & $32(100 \%)$ \\
\hline \multicolumn{2}{|l|}{ Disease status (RECIST 1.1) } \\
\hline SD & 16 \\
\hline PD & 15 \\
\hline
\end{tabular}

Continued 
Table 1 Continued.

\begin{tabular}{lcc}
\hline Demographics & & Values \\
\cline { 1 - 2 } Therapy & & \\
Surgery & $27(84 \%)$ \\
Chemotherapy & $2(6 \%)$ \\
PRRT & $4(13 \%)$ \\
SSA & $14(44 \%)^{\dagger}$ \\
No treated & $18(56 \%)^{\dagger}$ \\
\hline
\end{tabular}

\#Current therapy; *one also noradrenaline; **one also adrenaline and one dopamine; ${ }^{\dagger}$ current therapy.

$A$, adrenaline; BP, broncho-pulmonary; DA, dopamine; EBRT, external beam radiotherapy; GEP, gastroenteropancreatic; LCNEC, large-cell neuroendocrine carcinoma; NA, noradrenaline; NED, non-evidence of disease; PD, progressive disease; PRRT, peptide receptor radionuclide therapy; SCLC, small-cell lung cancer; SD, stable disease; SSA, somatostatin analogs.

was 34 years (range 12-62), and the gender distribution was M:F (17:15). The nine patients with more than one blood sample are summarized in Table 2 .

\section{Disease types}

Tumors were separated into three different groups: PGLs $(n=18)$, mixed PGL/PCC $(n=10)$ and PCCs $(n=4)$.

Table 2 Clinical details of PPGL $(n=9)$ with additional blood sampling.

\begin{tabular}{lc}
\hline Age, years (mean/range) & $35(21-62)$ \\
Gender (M:F) & $6: 3$ \\
Mutational status, $n$ & \\
SDHB: exon7, c.823G > T, p.R230L & 1 \\
SDHB: exon3, c. 402 C> T, p.R90X & 1 \\
SDHD: exon1, c.33 C>A, p. C11X & 6 \\
Functional status*, $n$ & \\
Non-secretor & 4 \\
Dopamine & 2 \\
Noradrenaline & 3 \\
Metastasis, $n$ & \\
Yes & 3 \\
No & 6 \\
Therapy during follow-up, $n$ & \\
SSA use & 3 \\
Surgery & 1 \\
No treatment & 5 \\
Disease status (RECIST 1.0), $n$ & \\
Baseline & \\
SD & \\
PD & \\
Follow-up blood & \\
SD $>$ SD & \\
SD $>$ PD & \\
PD $>$ SD & \\
PD $>$ PD & 2 \\
\hline
\end{tabular}

*amine, metabolite secretion; SD, stable disease; PD, progressive disease; SSA, somatostatin analogs.
Table 3 Mutation status in 31 of 32 patients (97\%).

\begin{tabular}{|c|c|c|}
\hline Syndrome & $n$ & Mutation \\
\hline PGL-1 & 19 & $\begin{array}{l}\text { SDHD: exon1, c.33 C>A, p. C11X }(n=18) \\
\text { SDHD: exon2, c. } 112 \text { C > T, p.R38X }(n=1)\end{array}$ \\
\hline PGL-3 & 1 & SDHC: exon 5, c.379 C> G, p.H127D \\
\hline PGL-4 & 5 & $\begin{array}{l}\text { SDHB: exon7, c.823G > T, p. R230L, }(n=1) \\
\text { SDHB exon3, c. } 402 \text { C > T, p.R90X, }(n=1) \\
\text { SDHB exon 7, c.784G }>\text { T, R217L, }(n=2) \\
\text { SDHB del.exon 1-2 }(n=1)\end{array}$ \\
\hline MEN2A & 1 & RET exon13, c.2372A > T, p. Y791F \\
\hline NF-1 & 3 & NF-1 \\
\hline VHL & 2 & VHL exon 1, c.451A>G, p.S80G \\
\hline
\end{tabular}

NF1 diagnosis was based on clinical features.

\section{Biochemical activity}

Seventeen (53\%) were biochemically inactive. Of the 15 biochemically active, seven secreted dopamine (one also noradrenaline) and 9 secreted noradrenaline (one also adrenaline and one also dopamine).

\section{Disease status}

In these cohorts, 1 patient exhibited no evidence of disease, 20 were considered stable (RECIST) and 11 exhibited disease progression. No radiological evidence of the disease was evident in the offspring $(\mathrm{F})$.

\section{Mutation status}

The mutation status was known in 31 of 32 (97\%) (Table 3).

\section{Biological clustering classification (29)}

Patients could be clustered into the two specific genetic mutational groups: Cluster 1 (SDHx/VHL) included 27 (84\%); Cluster 2 (RET/NF-1) included 4 (13\%), one patient could not be included due to an absence of genetic data.

\section{Comparator groups}

NETs: The demographics of the NET group are summarized (Table 1). Briefly, the majority of NETs were small intestinal $(n=19,59 \%)$, a preponderance $(n=23$, $72 \%$ ) were low grade (G1 or typical lung carcinoid) and $15(47 \%)$ were classified as progressive disease (RECIST). In terms of treatment, 18 were untreated and 14 (44\%) were receiving somatostatin analogs. No NETs were being treated with acid inhibitory therapy (PPIs) or anti-hypertensive medication. 
Controls: The control (non-NET) group included one IBD, three IPMN, 9 benign pancreatic cysts and 19 otherwise healthy controls. Eight individuals were taking PPIs. None of the controls were taking antihypertensive medication.

There were no differences in sex distribution: $\mathrm{M}: \mathrm{F}=17: 15$ (both groups) or age between PPGLs and either of the comparator groups (NETs: mean 38 years, range: 27-61; controls: median 38 years, range: 28-60; both $P=$ NS vs PPGLs, Mann-Whitney $U$ test) confirming the appropriateness of matching.

\section{Image analysis}

Morphological imaging (CT/MRI) was used to stage patients at study entry and to evaluate whether progression had occurred since earlier imaging $(n=9)$. RECIST 1.0 criteria were used to assess this.

\section{Blood sampling schedule}

Whole blood $(10 \mathrm{~mL})$ for transcript analysis was collected at baseline in 32 patients. Nine patients had a second blood draw 2-12 months thereafter (one individual had a third blood draw). Plasma for catecholamine and CgA analysis was obtained at exactly the same time points.

\section{PCR-based transcript analysis (NETest)}

\section{Technique}

The NETest assesses biological activity using gene inference technology and cancer hallmark prediction (18). Details of the PCR methodology, mathematical analysis and validation have been published in detail $(18,23,30,31)$. The procedure uses a 2-step protocol (RNA isolation, cDNA production and PCR) $(30,31)$ from EDTA-collected whole blood $(30,31)$. The expression of 51 NET marker genes includes the analysis of clusters of biologically relevant genes that constitute the different 'omes' (proliferome, metabolome, secretome, epigenome and pluromes) (18), which define the NET 'fingerprint'. Expression was normalized to housekeeping genes and quantified vs a population control (30).

\section{Biomathematics}

Multianalyte algorithm analysis (MAAA) was undertaken (SVM, LDA, KNN and Bayes) for categorization into different groups using 'majority vote' (30). This results in a $0-8$ score $(30,31)$, which is converted to an activity ranging from 0 (low activity) to $100 \%$ (high activity) based on the expression of 'omic' genes (18). Elevated expression of these genes is used to weight the score such that a high score e.g., ' 8 ' when combined with elevated 'omes' (identified to differentiate progressive from stable disease (18)) is scaled to $100 \%$ (high activity). A score of ' 8 ' with a low 'ome' is weighted to $53 \%$. We have determined the ranges that conform to clinical disease assessment in GEP-NETs: minimal activity: <0-14\%, low activity: 14-40\% and intermediate-high activity: >40-100\% (18).

Genes involved in proliferation, metabolism, epigenetics and somatostatin receptor expression

We also evaluated the gene expression in the proliferome, metabolome, epigenome and SSTRome. Genes in the proliferome (Ki67, NAP1L1 and NOL3) have wellcharacterized roles in GEP-NETs (Ki67 $(32,33,34)$ and NAP1L1 $(35,36,37))$ have well-described biological roles (NOL3 (38)). Genes in the metabolome (ATP6V1H, OAZ2, PANK2 and PLD3) are not as well characterized in GEPNETs, but their biological roles are well described: ATP6VIH regulates neuroendocrine oxidative phosphorylation (39), OAZ2 is involved in polyamine biosynthesis (40), PANK2 in metabolism and oxidation (41) and PLD3 in lipid metabolism and hypoxic signaling (42). Genes involved in the epigenome include MORF4L2, NAP1L1, PQBP1, RNF41, RSF1, SMARCD3 and ZFHX3. NAP1L1 has a well-defined role in GEP-NETs in the regulation of P53/KIP2 transcription through the regulation of promoter methylation (37). MORF4L2 is involved in neoplastic chromatin remodeling (43), PQBP1 regulates $\mathrm{X}$-chromosome transcription particularly during neural development (44), RNF41 is involved in regulating cellular differentiation (45), RSF1 is a well-characterized chromatin remodeler (46), SMARCD3 is a component of chromatin remodeling involved in epithelial-to-mesenchymal reprogramming (47) and $Z F H X 3$ is a transcriptional factor (48). Somatostatin receptor gene expression is a wellestablished feature of GEP-NETs (49) and of PPGLs (50).

Normalized gene expression for each of these 'omes' is presented. The upper limit of normal for the proliferome is 4.0; for the metabolome it is 2.4; for the epigenome it is 14.3 and for the SSTRome is 46.0 (18).

\section{Biochemical assays}

CgA was measured using the NEOLISA CgA kit (Euro Diagnostics, Malmo, Sweden) $(51,52)$. A cut-off 
of $108 \mathrm{ng} / \mathrm{mL}$ defined the upper limit of our normal population. Values $>108 \mathrm{ng} / \mathrm{mL}$ were considered as elevated. Plasma-free normetanephrine, metanephrine and methoxytyramine measurements were performed using the UltiMate 3000 HPLC system equipped with a Coulochem III detector (both from Thermo Fisher Scientific), according to a previously described HPLC-ECD method, for which lower limits of quantification had been established at $10 \mathrm{pg} / \mathrm{mL}$ for all the analytes of interest (53).

\section{Statistical analyses}

Analyses included Fisher's test (2-tailed), non-parametric (Mann-Whitney 2-tailed) measurements and receiveroperator curve (ROC) measurements. Regression analyses (uni and multiple) were undertaken to identify the significant clinical parameters that were independent risk factors for disease progression. Prism 6.0 for Windows (GraphPad Software, www.graphpad.com) and MedCalc Statistical Software, version 16.2.1 (MedCalc Software bvba, Ostend, Belgium; http://www.medcalc.org; 2013) were used. For individual biomarkers, the data are presented as mean \pm S.E.M. A $P$ value $<0.05$ was considered statistically significant.

\section{Results}

All healthy controls $(n=32)$ had normal NET transcript scores of $8 \pm 1.4 \%$. The GEP-NET comparator group was $100 \%$ positive and had scores of $46.8 \pm 4.7 \%$. The entire PPGL group who exhibited radiological evidence of disease $(n=30)$ had $100 \%$ NETest-positive blood samples with mean score values of $54.5 \pm 5 \%$. Both NET and PPGL scores were significantly higher than those in controls $(P<0.0001)$. Overall, NETest scores in PPGLs were similar to GEP-NETs $(P=0.37)$.

\section{Assessment of circulating transcript levels in PPGLs}

NETest scores were significantly $(P<0.0001)$ elevated in PPGLs and GEP-NETs compared to age- and sexmatched healthy controls (Fig. 1A). Median scores were $40 \%$ (PPGL) and 33\% (GEP-NET) vs 7\% (controls). No significant differences were identified between PPGLs and GEP-NETs. Values for all PPGLs $(n=32)$ ranged from $13 \%$ to $93 \%$. One patient $(3 \%)$, a 35 -year-old female (with a germline SDHD C11X mutation and no evidence of disease) had a score of $0 \%$. The AUC of the ROC analysis comparing PPGLs and controls was
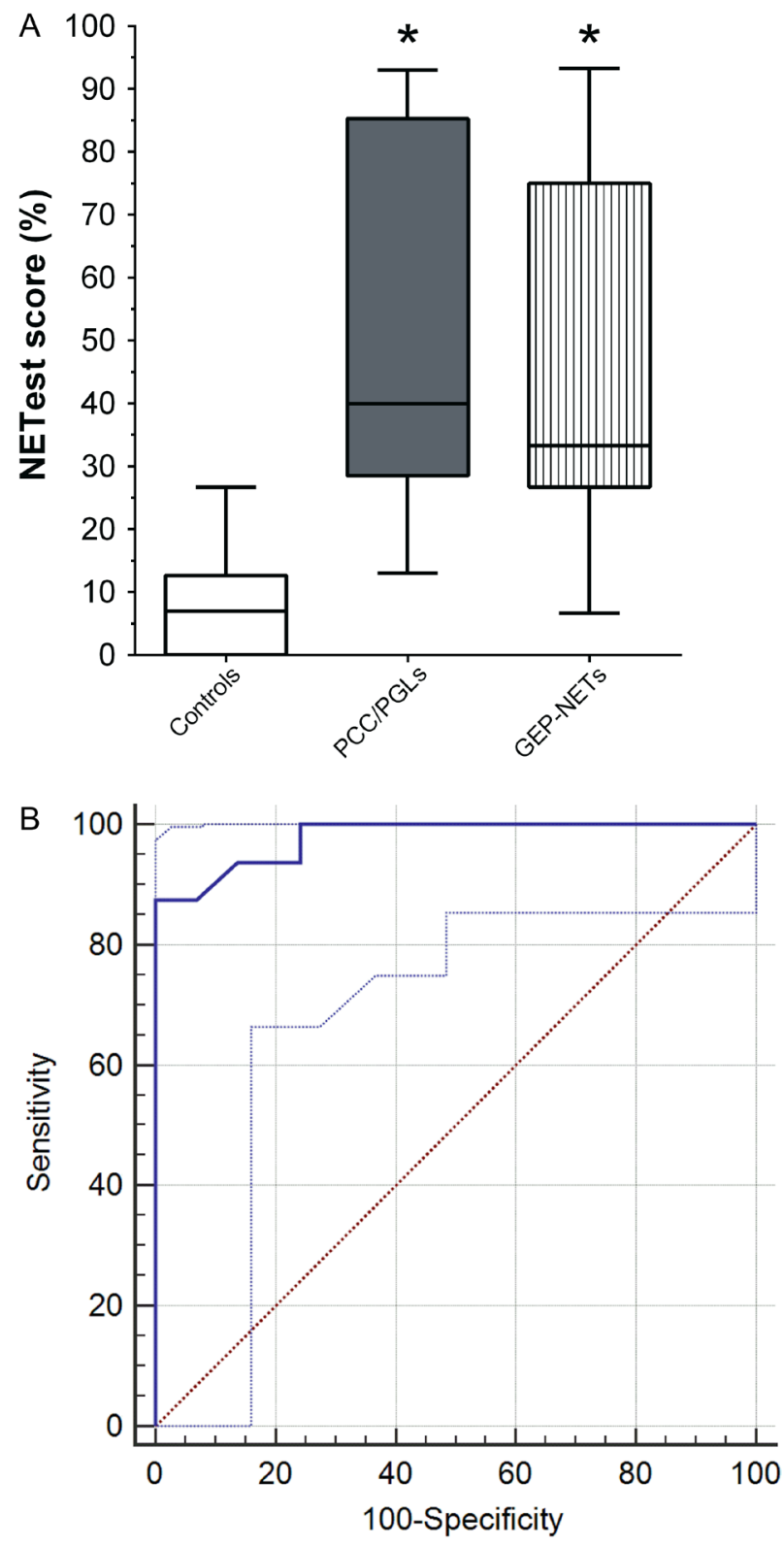

\section{Figure 1}

NETest in controls, GEP-NETs and PPGLs. (A) NETest scores were significantly elevated in PPGLs and GEP-NETs vs controls.

(B) ROC analysis identified that PPGLs could be differentiated from controls with an AUC of 0.98 . The Youden index ( () was 0.875 , and the optimal cut-off (sensitivity $87.5 \%$, specificity $100 \%$ ) for the NETest to differentiate PPGLs from controls was $>26.7 \%$. GEP-NETs, gastroenteropancreatic neuroendocrine tumors; PPGLS, pheochromocytomas/paragangliomas; ULN, upper limit of normal (14\%). ${ }^{*} P<0.0001$ vs controls (Mann-Whitney U-test). Blue dotted lines are the $95 \%$ confidence interval for the AUC (solid blue line, B). Red diagonal line is an AUC of $50 \%$ (B). 
$0.98 \pm 0.01$ (95\% CI: 0.95-1.01), $P<0.0001$ (Fig. 1B). The Youden index $(J)$ was 0.875 and the optimal cut-off for the NETest to differentiate PPGLs from controls in this series was $26.7 \%$. This value has been used in this study as the upper limit of normal (ULN).
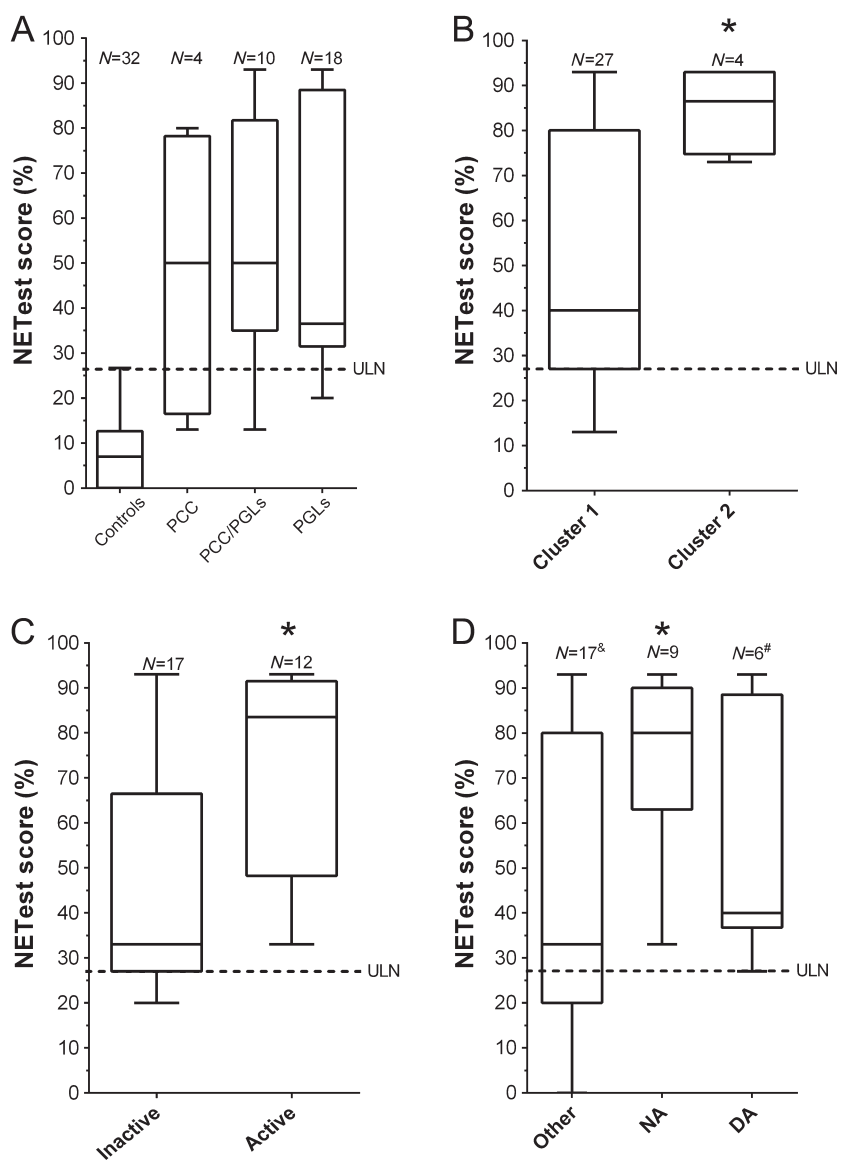

\section{Figure 2}

NETest in controls and PPGLs subtypes. (A) Distribution of NETest scores in different PPGL types compared to age-/sex-matched controls. Levels were elevated in all types (PCC, mixed PCC/PGLs and PGLs) vs controls. (B) NETest scores in Cluster 1 (SDHx/VHL) or Cluster 2 (RET/NF-1) tumors were significantly elevated in Cluster 2 tumors. (C) NETest scores in biochemically inactive and active (dopamine/noradrenalinesecreting) tumors were significantly elevated in secreting tumors. (D) NETest scores in noradrenaline secretors (NA), dopamine secretors (DA) and non-NA or DA secretors (other - the majority are non-secretors). Scores were significantly elevated in NA secretors. ULN, upper limit of normal $\left(26.7 \%\right.$ - this study). ${ }^{\star} P<0.04$ vs Cluster 1 (B). $* P<0.005$ vs inactive (Mann-Whitney $U$-test) (C). ${ }^{*} P<0.02$ vs other (D). "The one mixed NA/DA secretor was included in the NA group.

\section{NETest in PPGLs subtypes}

NETest scores in PCCs $(n=4)$ ranged from $13 \%$ to $80 \%$ (median 50\%) (Fig. 2A), whereas it ranged from 13\% to 93\% in the mixed PCC/PGL group $(n=10)$ with a median of $50 \%$. The PGL group ranged from $20 \%$ to $93 \%$ (median: $36.5 \%, n=18)$. All subtypes were significantly elevated vs controls, but scores were not different between subtypes.

An evaluation of the different mutational groups (SDHx: PGL-1, PGL-3 and PGL-4) and MEN2A, VHL and $N F-1$ identified that scores were not significantly different in any single mutational type (mean values: 40-55\%; $P=$ NS). NETest scores were, however, highest in those PGL-4 with detectable, progressive disease $(91 \pm 3.5 \%)$. NETest scores were significantly different when assessing tumors classified as Cluster 1 and Cluster 2 (Fig. 2B). Cluster 2 was associated with significantly higher scores $(84.8 \pm 5 \%$ vs $50.3 \pm 5 \%, P<0.04)$.

NET scores were also significantly different when tumors were classified by secretory status (biochemically inactive or active) (Fig. 2C). Secretory tumors had significantly higher scores than non-secretors $(73.3 \pm 6.5 \%$ vs $44.3 \pm 6 \%, P<0.005)$. A sub-analysis of the biochemically active cohort $(n=15)$, identified that noradrenaline secretors had the highest NETest levels $(74.7 \pm 6.7 \%)$, significantly higher than other tumors $(41.8 \pm 7.1 \%, P<0.02)$. This was not, however, different from dopamine secretors $(54.5 \pm 11.14 \%, P=0.26)$.

\section{NETest and PPGL disease status}

NETest scores were significantly higher in those with metastatic $(80 \pm 9 \%)$ and multicentric disease $(64 \pm 9 \%)$ than localized disease $(43 \pm 7 \%, P<0.05)$ (Fig. 3A). Disease-free patients (no radiological evidence of disease) had the lowest scores $(8.7 \pm 4.3 \%)$. A sub-analysis of the metastatic disease cohort identified that 2 of the $4(50 \%)$ were noradrenaline secretors.

Based upon imaging, eleven PPGLs were considered progressive, 19 stable and 3 (2 patients, one daughter with the mutation) disease free. The NETest scores were significantly $(P<0.0001)$ elevated in progressive disease $(86 \pm 2 \%)$ compared to stable disease $(41 \pm 5 \%)$ and disease free $(8.7 \pm 4.3 \%)$ (Fig. 3B). Significant differences were identified in the NETest between stable and disease free $(P<0.001)$. ROC analysis comparing progressive and stable disease identified an AUC of $0.93 \pm 0.05$ (95\% CI: 0.84 1.03), $P<0.0001$ (Fig. 3C). The Youden index was 0.875 and the optimal cut-off for the NETest to differentiate progressive disease from stable disease was $>53 \%$. 

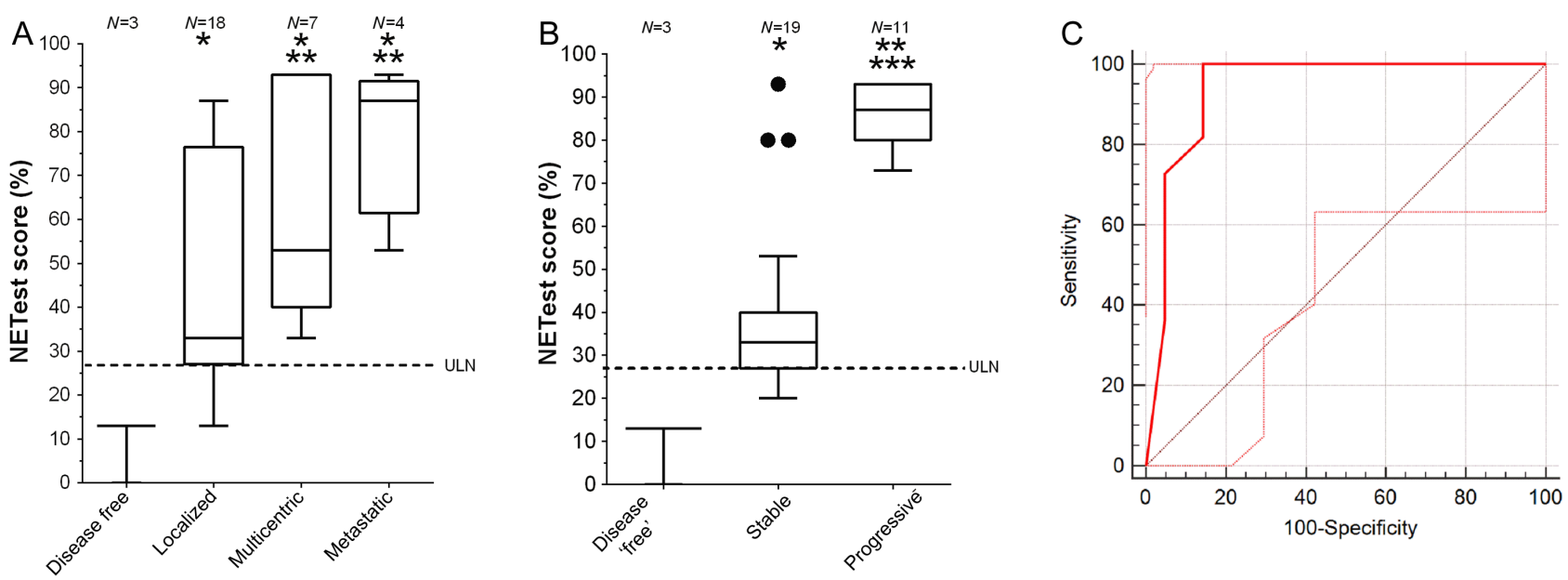

Figure 3

NETest in PPGLs based upon the extent or disease status. (A) NETest scores were significantly elevated in metastatic $(n=4)$ and multicentric disease $(n=13)$ vs localized $(n=13)$ and disease free $(n=3$, this includes the daughter of a patient with a known mutation and no disease). Two of the four metastatic disease patients were NA secretors. (B) NETest scores were significantly elevated in progressive disease than stable disease and disease-free patients. Zero of three disease-free patients secreted NA, four of 19 stable disease were NA+ and 5 (of 11) progressive disease were NA secretors. (C) ROC analysis demonstrated progressive PPGL disease could be differentiated with an AUC of 0.99 vs stable disease. The Youden index $(J)$ was 0.857 and the optimal cut-off (sensitivity $100 \%$, specificity $85.7 \%$ ) for the NETest to differentiate progressive disease from stable disease was $>53 \%$. ULN, upper limit of normal $\left(26.7 \%\right.$ - this study). ${ }^{*} P<0.0004$ vs disease-free; ${ }^{*} P<0.02$ vs localized. ${ }^{*} P<0.0001$ vs disease-free; $* * P<0.001$ vs stable; $* * * P<0.003$ vs disease free. Red dotted lines are the $95 \%$ confidence interval for the AUC (solid red line, B). Red diagonal line is an AUC of $50 \%$ (B).

An assessment of the secretory status identified that proportionally more noradrenaline secretors were associated with progressive disease (5 of $11,45 \%$ ) than stable disease (4 of 19, 21\%). This was, however, not statistically significant (Fisher's test: $P=0.7$ ).

\section{NETest genes involved in PPGL disease progression}

Progressive disease in GEP-NETs is linked to tumor gene expression that regulates proliferation, metabolism and the epigenome and also includes some secretory genes as well as genes involved in pluripotency and somatostatin receptor expression (18). We evaluated the gene expression in each of these 6 'omes' in the PPGL cohort. Specifically, we assessed whether a similar pattern of expression occurred as in GEP-NETs. An analysis of stable $(n=19)$ and progressive $(n=11)$ PPGL disease groups identified that circulating proliferation-associated gene expression was significantly $(P<0.0001)$ elevated in the progressive disease cohort $(36.8 \pm 6.8$ vs $10.5 \pm 1.2$, Fig. 4A). In addition, the epigenome (PD: $24.3 \pm 6.5$ vs SD: $11.2 \pm 2.1, P<0.03$ ) and SSTRome (PD: $133 \pm 40$ vs SD: $53 \pm 10, P<0.001)$ were also significantly elevated.
Metabolomic expression was, however, significantly reduced $(0.47 \pm 0.19$ vs $3.1 \pm 0.6, P<0.04)$.

Gene expression linked to metabolism was also identified to be significantly $(P<0.003)$ reduced in PPGLs as a group $(2.1 \pm 0.5)$ compared to GEP-NETs as a group $(3.3 \pm 0.6)$ (Fig. 4B). This was particularly evident in those with $S D H x$ mutations $(0.9 \pm 0.2$ vs $5.5 \pm 1.3, P<0.0001$, Fig. 4C). A sub-analysis of progressive and stable disease identified that metabolome expression in progressive disease, consistent with $S D H x$ mutations, was lower than those with stable disease $(0.9 \pm 0.3$ vs $3.6 \pm 1.5, P=0.06)$.

\section{Amine measurements and NETest}

Circulating catecholamine measurements demonstrated that in subjects with detectable disease $(n=30)$, plasma free methoxytyramine was elevated in 23\% $(n=7)$, plasma free normetanephrine in 30\% $(n=9)$ and CgA in $27 \%(n=8)$. Two patients also had elevated plasma free metanephrines. In contrast, NETest score was positive in all 30 individuals (100\%) with image-detectable PPGLs disease (Fig. 5). This difference was statistically significant (Fisher's exact test: $P<0.0001)$. 


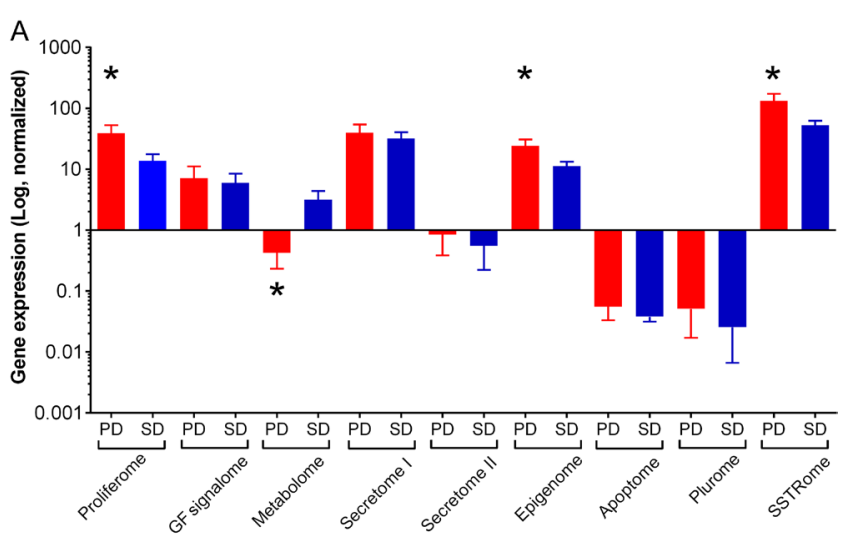

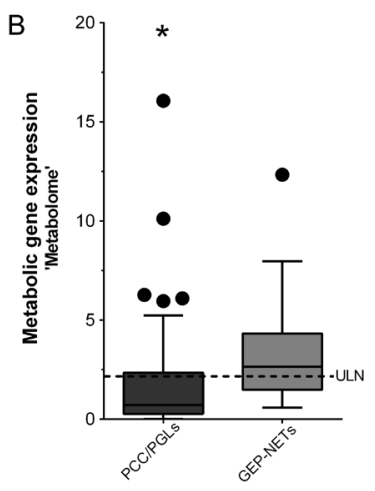

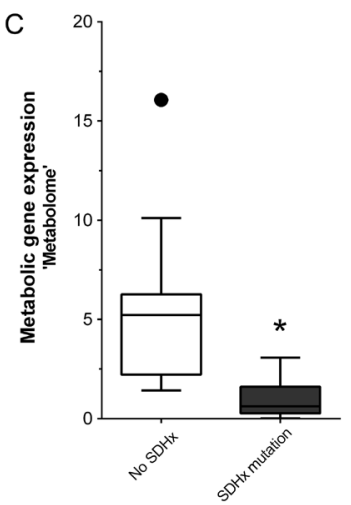

Figure 4

Proliferome and metabolome gene expression. (A) Omic gene expression in progressive disease (PD) and stable disease in PPGL. Significantly elevated gene expression in PD was identified for the proliferation, epigenetics and somatostatin receptors, whereas genes involved in regulating metabolism were decreased. (B) Box and whisker plot (Tukey) showing median, 25th and 75th percentiles and range of metabolome gene expression in PPGLs and GEP-NETs. Levels were significantly decreased in PPGLs. (C) Sub-analysis of metabolome gene expression in the PPGL group identified that this was significantly decreased in PPGLs with SDHx mutations $(n=25)$ compared to other mutation types $(n=7)$. ULN, upper limit of normal (proliferome: 4, metabolome: 2.4). ${ }^{*} P<0.04$ vs stable disease (A). ${ }^{*} P<0.001$ vs GEP-NETs (B). $* P<0.001$ vs no SDHx mutation (C).

\section{Multivariate assessment of biomarkers and clinical information and disease progression}

The Ki-67 index and tumor size/TNM classification have previously been associated with progressive disease $(54,55)$. In our series, however, Ki-67 was available in only 13 patients (41\%), the majority $(12,92 \%)$ of whom had a labeling index $<2 \%$. TNM classification was only available

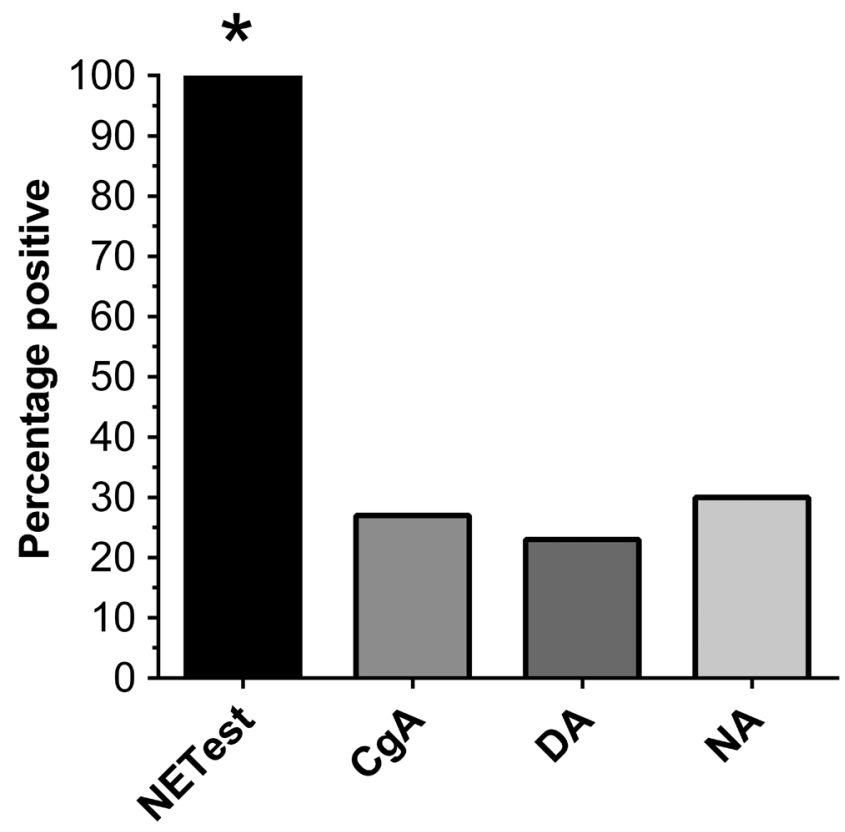

Circulating biomarker

\section{Figure 5}

Circulating biomarker levels. NETest was positive in all disease cases. Overall, single analyte biomarkers were detectable in $47 \%$ of cases. Individually, levels were elevated in $6-28 \%$. ${ }^{*} P<0.001$ vs single analytes (Fisher's exact text). $\mathrm{CgA}$, chromogranin $\mathrm{A}$; DA, dopamine (plasma free methoxytyramine); NA, noradrenaline (plasma free normetanephrine).

in 15 (47\%; those who had had surgery), the majority of whom were pT1 (67\%). The size/weight of the tumors was also therefore not available. Given that $<50 \%$ of patients had these data, we did not include this information in the univariate or multivariate analyses.

Univariate analysis of clinical parameters and standard biomarkers at baseline identified that no parameter was associated with disease progression. Neither cluster type (F-ratio: $0.63, P=0.43$ ) nor $S D H B$ mutation ( $F: 0.08, P=0.78)$ were linked to progression. Secretion $(F: 1.88, P=0.18)$ and especially noradrenaline secretion $(F: 2.53, P=0.12)$ exhibited a trend toward an association, but this was not statistically significant. Metastasis too showed a trend $(F: 1.71, P=0.20)$. The only significant variable in univariate analysis was a NETest score $>53 \%$ ( $F$ : 61.87, $P<0.0001$ ). To confirm this, we undertook a multivariate analysis with these six factors. The only significant factor in analysis was the elevated NETest score (coefficient $0.77 \pm 0.12, r_{\text {partial }}=0.78$, $P<0.0001$; Table 4). 
Table 4 Multivariate analysis of parameters that predict progressive disease at baseline $(n=32)$.

\begin{tabular}{lll}
\hline Independent variables & & Coefficient \\
\cline { 1 - 1 } (Constant) & & -0.04822 \\
Cluster type & & -0.002459 \\
Metastasis & 0.1835 \\
Noradrenaline secretion & & -0.1527 \\
NETest $>53 \%$ & & $\mathbf{0 . 7 7 0 6}$ \\
SDHB mutation & & 0.04511 \\
Secretory phenotype & 0.1196 \\
\hline
\end{tabular}

\section{Clinical utility of the NETest}

Nine patients had two blood samples (one had three sample collections) over a 12-month period (Fig. 6). At baseline, 3 (33\%) exhibited progressive disease. One patient was not treated and the disease continued to progress. A second patient was treated with somatostatin analogs and over a two-month period exhibited a decrease in the NETest to 33\%. Image-based assessment (CT and somatostatin receptor imaging/SRI) at this time identified disease stabilization. The third patient underwent curative
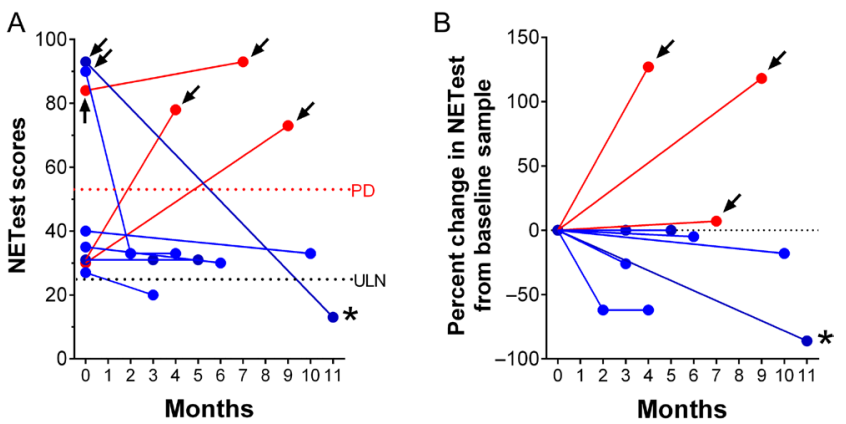

\section{Figure 6}

NETest levels over time in nine patients. (A) Actual NETest measurements. At baseline (time T0), 3 of the cohort exhibited clinical evidence for progressive disease (arrows). Over time, one patient continued to progress, and two patients developed progressive disease. Two of the 3 with PD at baseline underwent either surgery (now no evidence of disease) or responded well to a somatostatin analog. Four patients were stable for the duration. (B) Spider plot identifying the changes from baseline. All three patients who continued to progress or developed progressive disease (arrows) exhibited a positive change in the NETest score. Those who were stable or underwent curative surgery demonstrated a decrease in scores. ULN, 26.7\% (identified from this study). PD, 53\% (differentiates stable from progressive disease - this study). Arrows identify scores associated with clinically confirmed progressive disease. Star identifies patient who underwent surgery. surgery. The NETest measured at 11 months was identified to be $13 \%$. This falls within the normal range and was consistent with imaging (CT/SRI), which did not identify residual disease. Two of the six patients with stable disease at baseline developed progressive disease. One had a noradrenaline-secreting tumor characterized by a SDHD mutation (c.33 C/A C11X, Ki67: 1\%), the second had a non-secreting tumor (same mutation, Ki67: 2\%). Both had significant increases in the NETest of 125-127\% from baseline (Fig. 6B). The four stable patients who remained stable over the course of follow-up (3-10) months all exhibited NETest scores $<40 \%$ that were either the same (unchanged - Fig. 6B) or dropped $(n=2)$, consistent with a response to therapy (both on SSAs).

\section{Discussion}

The precise clinical and biological assessment of PPGLs has proved difficult as descriptive histological approaches, complex imaging appearances and measurements of circulating biomarkers have all exhibited some degree of limitation in clinical utility (56). Newer approaches including molecular genetics $(4,29)$ have begun to advance the elucidation of this group of tumors and facilitate classification. In particular, the identification of canonical molecular pathways that are altered by disease has provided information that has facilitated the identification of disease progression. We have previously developed circulating mRNA methodology that has diagnostic relevance as well as providing information informative of the biological behavior of GEP-NET $(18,30)$. In this investigation, we sought to apply this methodology to the assessment of a cohort of welldifferentiated PPGL neuroendocrine tumors.

We demonstrated that the NET-based circulating signature (mRNAs) could effectively (100\%) detect both well-differentiated PGLs and PCCs, that levels of gene expression could differentiate benign from malignant PPGL disease in this cohort, that the signature was effective (i.e., positive) in both secreting as well as non-secreting 
lesions and that specific elements of the signature that capture neoplastic hallmarks (17), namely that 'omic analysis' of the proliferation, epigenetics, somatostatin receptors and metabolism-related gene clusters, correlated with disease activity (both at baseline and follow-up) and mutational status (Cluster 2 vs Cluster 1). Moreover, NETest score $>53 \%$ was identified as an independent predictor of disease progression. These data, taken as a group, indicate that the multianalyte neuroendocrine gene transcript analysis can be used as a clinical marker for well-differentiated PPGL gene expression, is associated with the underlying mutational status and provides a biologically and clinically accurate tool for defining disease activity.

Study strengths of this investigation include that it was undertaken in a 'real-world' clinical setting with standardized imaging and biomarker assessment, that age- and gender-matched controls and GEP/BP-NETs were used as a comparator and that the PPGL mutational status was known. Limitations of the study include the relatively small numbers (32 PPGLs) evaluated, that the cohort was not homogeneously treated (and were at different disease stages), that the majority were characterized by $S D H x$ mutations, that few PCCs were included and that no poorly differentiated PPGLs were studied.

PPGLs are principally hereditary-driven tumors whose susceptibility has been coupled to at least 15 different genes (29). Germline mutations are associated with $\sim 40 \%$ of all cases, whereas somatic mutations in susceptibility genes account for about a third of sporadic cases (29). Transcriptomic studies by others have identified the two principal molecular pathways (Cluster 1 and Cluster 2) associated with these tumors (29). The hypoxic pathway is activated in Cluster 1, whereas MAPK and mTOR (mammalian target of rapamycin) signaling pathways are activated in Cluster 2. When this is coupled with biochemical data (57), Cluster 1 includes tumors with mutations in SDHx, VHL and HIF2A that typically secrete norepinephrine and/or dopamine $(4,5)$. Cluster 2 includes tumors with mutations in RET, NF-1, TMEM123 and MAX (4). These are typically epinephrine or mixed epinephrine/ norepinephrine-secreting tumors (5). This 'omic' approach has served as a novel classification protocol and provided a tool that has utility in the identification of new therapeutic and potentially diagnostic targets (56). Well-differentiated PPGLs and GEP-NETs share a number of common mutations. GEP-NETs are associated with germline or sporadic mutations in $S D H B, V H L$ and $N F-1$ $(24,25)$, as well as in the mTOR/AKT pathways $(58,59)$ and exhibit common downstream transcriptional and pathway abnormalities (e.g., hypoxia signaling, RAF/RAS activation) (24). As PPGLs are considered components of the neuroendocrine tumor 'family', it is conceivable that diagnostic targets identified in GEP-NETs may be relevant to well-differentiated PPGLs.

The molecular signature of neuroendocrine tumor disease (NETest) was detectable in all (100\%) PPGLs (both PCCs, mixed PCC/PGL and PGLs) with clinical and imaging evidence of disease. Disease-free patients had scores that were indistinguishable from controls. A ROC analysis score identified the test to be $>95 \%$ positive for differentiating PPGLs from controls. This value exceeds the standard accepted cut-off of $80 \%$ for an effective diagnostic test (60).

GEP-NETs, with progressive disease or greater tumor burden, are associated with significantly higher scores $(22,23)$. The same holds for PPGLs in this study. Thus, increased gene expression scores were associated with increased tumor burden. The lowest NETest scores were identified in localized HNPs and significantly higher scores were evident in multicentric and metastatic PPGL disease. The latter typically exhibited progressive disease. This reflects the significant overexpression of proliferationassociated genes as well as mRNA involved in epigenetic regulation and somatostatin receptor expression. These data are concordant with similar observations in GEPNETs (18). Interestingly, however, PPGLs did not express elevated expression of genes involved in growth factor signaling, which is likely related to the two very different growth milieu, i.e., bowel vs neural ganglia. Moreover, the cut-off for detecting progression was lower in PPGLs $(>53 \%$, current study) than that published for GEP-NETs $(80 \%)(22,23)$. As the index is based on the addition of normalized gene expression, it is not surprising that this is lower in PPGLs that are characterized by fewer progressive disease-associated 'omes'. Irrespective, the NETest was the only independent prognostic factor identified in the study.

Although the mutational status of the tumor was not directly reflected in NETest scores the use of cluster grouping (Cluster 1 or Cluster 2) indicated that NETest scores were higher in Cluster 2, the non-SDHx/VHL mutation group. This is consistent with our previous observations that the NETest is elevated in malignant and progressive GEP-NETS $(22,23)$. Thus, in well-differentiated PPGLs, it appears that the circulating transcripts identify a malignant phenotype associated with these mutations and presumably define some aspects of the biological behavior (activation of kinase signaling pathways) of the tumor associated with its malignant propensity 
$(29,56)$. In addition, scores very effectively differentiated progressive from stable disease (ROC: 0.93) suggesting that circulating mRNAs can provide accurate, real-time information about tumor biology and disease activity.

SDHx mutations were associated with decreased expression of metabolism-associated genes in PPGLs. The overall group was predominantly $S D H x$ mutated (78\%) who exhibited lower metabolism gene expression values than those with Cluster 2 mutations (NF-1 and MEN2A). The correlation identified in this study between mutation and metabolic gene expression in PPGLs suggests that circulating neuroendocrine mRNA levels can be used to provide further insight into the biological behavior of PPGLs. In comparison, the higher levels of gene expression in GEP-NETs likely reflect the low level of SDHD mutations in this group (61).

Pheochromocytomas, like PGLs, were also positive using the NETest, indicating that the multianalyte gene approach can detect this tumor subset. The relatively small numbers of pheochromocytomas in the current study $(n=3)$, however, limited interpretation. Nevertheless, the one PCC with an elevated score of $93 \%$ was a 39 -year-old male with familial MEN2A (RET p. Y791F, noradrenaline secretion and Ki67: 2\%), with progressive disease. As a total thyroidectomy had been previously undertaken for medullary thyroid carcinoma and thyrocalcitonin levels were normal, we infer that the elevated score reflected transcript activity of the PCC.

Until recently, liquid biopsy approaches to PPGLs have been lacking with a focus on tissue-based strategies. In this respect, a Ki67 index $>3 \%$ has been suggested as a predictor of tumor behavior $(54,55)$. This is, however, not an invariant marker (62). As an alternative, two scoring systems (GAPP (7) and PASS (8)) have been developed to predict the behavior. Neither, however, has been definitively validated and both use histomorphology (a subjective assessment) rather than molecular or functional biology. The GAPP system is a combination of histological pattern, cellularity, necrosis, capsular/ vascular invasion, Ki67 labeling index and catecholamine immunohistochemistry. The output is a $0-10$ scale that divides tumors into three groups - low, intermediate or high (7). Although GAPP scores generally exhibit some correlation with the two recently proposed tumor clusters and has some utility for differentiating nonmetastatic and metastatic groups (7), concerns have been raised regarding its clinical utility (absence of genetic information) (57). Biomarker-based assessments, likewise, are limited by a range of issues including reliability, variability and standardization and have diminished or limited value when tumors are non-secretory. Functional tumors may be associated with life-threatening complications (hypertension) (63). In the current study, $53 \%$ of tumors were classified as non-functional. About $50 \%$ of functional tumors secreted CgA, of these, secretory biomarkers (adrenaline and noradrenaline) and metabolic products (normetanephrine, metanephrine and methoxytyramine), were only detectable in $10-60 \%$ of the cases. In all lesions irrespective of tumor secretory status or functionality (that were biomarker negative), the measurement of circulating neuroendocrine mRNA transcripts were positive. NETest scores were significantly elevated in biochemically active tumors. In this cohort, tumors tended to be more aggressive ( 8 of the 11 progressive disease cohorts were biochemically active) and circulating transcripts provided further information in the identification of disease activity. Interestingly in our cohort, noradrenaline secretion appeared to be a secretory marker that might be associated with progressive disease. This is consistent with an earlier retrospective analysis (64). A multivariate approach, however, did not confirm the utility of this marker. The latter finding is supported by a separate study identifying noradrenaline overexpression in more benign tumors (65).

These results support previous observations that multianalyte approaches that capture fundamental tumor biology (66) are significantly more effective than individual measurements of circulating monoanalyte secretory amines or peptides in disease identification. In addition, they provide a rationale for measuring biologically relevant gene expression that may provide added information regarding the underlying mutational status of a specific tumor. This knowledge may be used to better define the clinical status of the individual's disease and thereby facilitate the management.

Transcript analysis (NETest) is positive in welldifferentiated PPGLs and mRNA expression levels correlate with tumor burden. Furthermore, expression levels correlate with disease activity (malignancy), and proliferation-, epigenetic- and SSTRome-associated genes are elevated in progressive disease. It seems likely that evaluation of these as well as metabolic gene expression may provide useful information in assessing the biological basis of disease activity in PPGLs. Of note is the observation that the NETest is positive when other circulating biomarkers are negative and that elevated levels $(>53 \%)$ are an independent predictor of disease progression. Overall, our studies support Favier et al.'s proposition that an 'omic' approach for diagnosis and treatment of well-differentiated PPGLs is feasible and of 
clinical utility (29). The data we have presented require validation in a larger cohort as well as evaluation in poorly differentiated PPGLs. They do, however, in principle, support the proposal that a blood-based tool measuring tumor transcripts is practical and suggest that such a strategy has biological relevance and clinical utility.

\section{Declaration of interest}

The authors declare that there is no conflict of interest that could be perceived as prejudicing the impartiality of the research reported.

Funding

The study was supported by Clifton Life Sciences.

\section{Author contribution statement}

All authors contributed equally to the manuscript.

\section{References}

1 Lenders JW, Duh QY, Eisenhofer G, Gimenez-Roqueplo AP, Grebe SK, Murad MH, Naruse M, Pacak K \& Young WF Jr. Pheochromocytoma and paraganglioma: an endocrine society clinical practice guideline. Journal of Clinical Endocrinology and Metabolism 201499 1915-1942. (doi:10.1210/jc.2014-1498)

2 Fishbein L, Orlowski R \& Cohen D. Pheochromocytoma/ paraganglioma: review of perioperative management of blood pressure and update on genetic mutations associated with pheochromocytoma. Journal of Clinical Hypertension 201315 428-434. (doi:10.1111/jch.12084)

3 Chen H, Sippel RS, O'Dorisio MS, Vinik AI, Lloyd RV, Pacak K \& North American Neuroendocrine Tumor Society. The North American Neuroendocrine Tumor Society consensus guideline for the diagnosis and management of neuroendocrine tumors: pheochromocytoma, paraganglioma, and medullary thyroid cancer. Pancreas 201039 775-783. (doi:10.1097/MPA.0b013e3181ebb4f0)

4 King KS \& Pacak K. Familial pheochromocytomas and paragangliomas. Molecular and Cellular Endocrinology 2014386 92-100. (doi:10.1016/j.mce.2013.1007.1032)

5 Dahia PL. Pheochromocytoma and paraganglioma pathogenesis: learning from genetic heterogeneity. Nature Reviews Cancer 201414 108-119. (doi:10.1038/nrc3648)

6 Neumann HP, Pawlu C, Peczkowska M, Bausch B, McWhinney SR, Muresan M, Buchta M, Franke G, Klisch J, Bley TA et al. Distinct clinical features of paraganglioma syndromes associated with SDHB and SDHD gene mutations. JAMA 2004292 943-951. (doi:10.1001/ jama.292.8.943)

7 Kimura N, Takayanagi R, Takizawa N, Itagaki E, Katabami T, Kakoi N, Rakugi H, Ikeda Y, Tanabe A, Nigawara T et al. Pathological grading for predicting metastasis in phaeochromocytoma and paraganglioma. Endocrine-Related Cancer 201421 405-414. (doi:10.1530/ERC-13-0494)

8 Thompson LD. Pheochromocytoma of the Adrenal gland Scaled Score (PASS) to separate benign from malignant neoplasms: a clinicopathologic and immunophenotypic study of 100 cases. American Journal of Surgical Pathology 200226 551-566. (doi:10.1097/00000478-200205000-00002)

9 Wu D, Tischler AS, Lloyd RV, DeLellis RA, de Krijger R, van Nederveen F \& Nose V. Observer variation in the application of the Pheochromocytoma of the Adrenal Gland Scaled Score. American
Journal of Surgical Pathology 200933 599-608. (doi:10.1097/ pas.0b013e318190d12e)

10 Darr R, Pamporaki C, Peitzsch M, Miehle K, Prejbisz A, Peczkowska M, Weismann D, Beuschlein F, Sinnott R, Bornstein SR et al. Biochemical diagnosis of phaeochromocytoma using plasma-free normetanephrine, metanephrine and methoxytyramine: importance of supine sampling under fasting conditions. Clinical Endocrinology 201480 478-486. (doi:10.1111/cen.12327)

11 Eisenhofer G \& Tischler AS. Neuroendocrine cancer. Closing the GAPP on predicting metastases. Nature Reviews Endocrinology 201410 315-316. (doi:10.1038/nrendo.2014.41)

12 van Berkel A, Lenders JW \& Timmers HJ. Diagnosis of endocrine disease: biochemical diagnosis of phaeochromocytoma and paraganglioma. European Journal of Endocrinology 2014170 R109-R119. (doi:10.1530/EJE-13-0882)

13 Olson SW, Yoon S, Baker T, Prince LK, Oliver D \& Abbott KC. Longitudinal plasma metanephrines preceding pheochromocytoma diagnosis: a retrospective case-control serum repository study. European Journal of Endocrinology 2016174 289-295. (doi:10.1530/EJE15-0651)

14 Eisenhofer G \& Peitzsch M. Laboratory evaluation of pheochromocytoma and paraganglioma. Clinical Chemistry 201460 1486-1499. (doi:10.1373/clinchem.2014.224832)

15 Mason CE, Porter SG \& Smith TM. Characterizing multi-omic data in systems biology. Advances in Experimental Medicine and Biology 2014 799 15-38. (doi:10.1007/1978-1001-4614-8778-1004_1002)

16 Izmailov R, Guest PC, Bahn S \& Schwarz E. Algorithm development for diagnostic biomarker assays. International Review of Neurobiology 2011101 279-298. (doi:10.1016/B1978-1010-1012-387718387715.300011-387710)

17 Hanahan D \& Weinberg RA. Hallmarks of cancer: the next generation. Cell 2011144 646-674. (doi:10.1016/j.cell.2011.02.013)

18 Kidd M, Drozdov I \& Modlin I. Blood and tissue neuroendocrine tumor gene cluster analysis correlate, define hallmarks and predict disease status. Endocrine-Related Cancer 201522 561-575. (doi:10.1530/ERC-15-0092)

19 Walenkamp A, Crespo G, Fierro Maya F, Fossmark R, Igaz P, Rinke A, Tamagno G, Vitale G, Oberg K \& Meyer T. Hallmarks of gastrointestinal neuroendocrine tumours: implications for treatment. Endocrine-Related Cancer 201421 R445-R460. (doi:10.1530/ERC-140106)

20 Li SC, Essaghir A, Martijn C, Lloyd RV, Demoulin JB, Oberg K \& Giandomenico V. Global microRNA profiling of well-differentiated small intestinal neuroendocrine tumors. Modern Pathology 201326 685-696. (doi:10.1038/modpathol.2012.216)

21 Modlin IM, Frilling A, Salem RR, Alaimo D, Drymousis P, Wasan HS, Callahan S, Faiz O, Weng L, Teixeira $\mathrm{N}$ et al. Blood measurement of neuroendocrine gene transcripts defines the effectiveness of operative resection and ablation strategies. Surgery 2016159 336-347. (doi:10.1016/j.surg.2015.1006.1056)

22 Pavel M, Jann H, Prasad V, Drozdov I, Modlin IM \& Kidd M. NET blood transcript analysis defines the crossing of the clinical rubicon: when stable disease becomes progressive. Neuroendocrinology 201615 15. (doi:10.1159/000446025)

23 Cwikla JB, Bodei L, Kolasinska-Cwikla A, Sankowski A, Modlin IM $\&$ Kidd M. Circulating transcript analysis (netest) in gep-nets treated with somatostatin analogs defines therapy. Journal of Clinical Endocrinology and Metabolism 2015 8. (doi:10.1210/jc.2015-2792)

24 Kidd M, Modlin I \& Oberg K. Towards a new classification of gastroenteropancreatic neuroendocrine neoplasms. Nature Reviews Clinical Oncology 201613 691-705. (doi:10.1038/nrclinonc.2016.85)

25 Zikusoka MN, Kidd M, Eick G, Latich I \& Modlin IM. The molecular genetics of gastroenteropancreatic neuroendocrine tumors. Cancer 2005104 2292-2309. (doi:10.1002/cncr.21451)

26 Kidd M, Modlin I, Bodei L \& Drozdov I. Decoding the molecular and mutational ambiguities of gastroenteropancreatic neuroendocrine 
neoplasm pathobiology. Cellular and Molecular Gastroenterology and Hepatology 20151 131-153. (doi:10.1016/j.jcmgh.2014.12.008)

27 Peczkowska M, Erlic Z, Hoffmann MM, Furmanek M, Cwikla J, Kubaszek A, Prejbisz A, Szutkowski Z, Kawecki A, Chojnowski K et al. Impact of screening kindreds for SDHD p.Cys11X as a common mutation associated with paraganglioma syndrome type 1. Journal of Clinical Endocrinology and Metabolism 200893 4818-4825. (doi:10.1210/jc.2008-1290)

28 Grossrubatscher E, Dalino P, Vignati F, Gambacorta M, Pugliese R, Boniardi M, Rossetti O, Marocchi A, Bertuzzi M \& Loli P. The role of chromogranin A in the management of patients with phaeochromocytoma. Clinical Endocrinology 200665 287-293. (doi:10.1111/j.1365-2265.2006.02591.x)

29 Favier J, Amar L \& Gimenez-Roqueplo AP. Paraganglioma and phaeochromocytoma: from genetics to personalized medicine. Nature Reviews Endocrinology 201511 101-111. (doi:10.1038/ nrendo.2014.188)

30 Modlin I, Drozdov I \& Kidd M. The identification of gut neuroendocrine tumor disease by multiple synchronous transcript analysis in blood. PLOS ONE 20138 e63364. (doi:10.1371/journal. pone.0063364)

31 Modlin I, Drozdov I \& Kidd M. Gut neuroendocrine tumor blood qpcr fingerprint assay: characteristics and reproducibility. Clinical Chemistry 201452 419-429. (doi:10.1515/cclm-2013-0496)

32 Foltyn W, Zajecki W, Marek B, Kajdaniuk D, Sieminska L, Zemczak A $\&$ Kos-Kudla B. The value of the Ki-67 proliferation marker as a prognostic factor in gastroenteropancreatic neuroendocrine tumours. Endokrynologia Polska 201263 362-366.

33 Yang Z, Tang LH \& Klimstra DS. Effect of tumor heterogeneity on the assessment of Ki67 labeling index in well-differentiated neuroendocrine tumors metastatic to the liver: implications for prognostic stratification. American Journal of Surgical Pathology 2011 35 853-860. (doi:10.1097/PAS.0b013e31821a0696)

34 Klimstra DS, Modlin IR, Adsay NV, Chetty R, Deshpande V, Gonen M, Jensen RT, Kidd M, Kulke MH, Lloyd RV et al. Pathology reporting of neuroendocrine tumors: application of the Delphic consensus process to the development of a minimum pathology data set. American Journal of Surgical Pathology 201034 300-313. (doi:10.1097/PAS.0b013e3181ce1447)

35 Drozdov I, Kidd M, Nadler B, Camp RL, Mane SM, Hauso O, Gustafsson BI \& Modlin IM. Predicting neuroendocrine tumor (carcinoid) neoplasia using gene expression profiling and supervised machine learning. Cancer 2009115 1638-1650. (doi:10.1002/ cncr.24180)

36 Kidd M, Modlin IM, Mane SM, Camp RL, Eick G \& Latich I. The role of genetic markers - NAP1L1, MAGE-D2, and MTA1 - in defining small-intestinal carcinoid neoplasia. Annals of Surgical Oncology 2006 13 253-262. (doi:10.1245/ASO.2006.12.011)

37 Schimmack S, Taylor A, Lawrence B, Alaimo D, SchmitzWinnenthal H, Buchler MW, Modlin IM \& Kidd M. A mechanistic role for the chromatin modulator, NAP1L1, in pancreatic neuroendocrine neoplasm proliferation and metastases. Epigenetics and Chromatin 20147 15. (doi:10.1186/1756-8935-1187-1115. eCollection 2014)

38 Sohn EJ, Shin MJ, Eum WS, Kim DW, Yong JI, Ryu EJ, Park JH, Cho SB, Cha HJ, Kim SJ et al. Tat-NOL3 protects against hippocampal neuronal cell death induced by oxidative stress through the regulation of apoptotic pathways. International Journal of Molecular Medicine 201638 225-235. (doi:10.3892/ijmm.2016.2596)

39 Olsson AH, Yang BT, Hall E, Taneera J, Salehi A, Nitert MD \& Ling C. Decreased expression of genes involved in oxidative phosphorylation in human pancreatic islets from patients with type 2 diabetes. European Journal of Endocrinology 2011165 589-595. (doi:10.1530/EJE11-0282)

40 Ruan Y, Cheng M \& Ou Y. Oko R \& van der Hoorn FA. Ornithine decarboxylase antizyme Oaz3 modulates protein phosphatase activity.
Journal of Biological Chemistry 2011286 29417-29427. (doi:10.1074/ jbc.M111.274647)

41 Hayflick SJ. Defective pantothenate metabolism and neurodegeneration. Biochemical Society Transactions 201442 1063-1068. (doi:10.1042/BST20140098)

42 Valli A, Rodriguez M, Moutsianas L, Fischer R, Fedele V, Huang HL, Van Stiphout R, Jones D, McCarthy M, Vinaxia M et al. Hypoxia induces a lipogenic cancer cell phenotype via HIF1alpha-dependent and -independent pathways. Oncotarget 20156 1920-1941. (doi:10.18632/oncotarget.3058)

43 Shadeo A, Chari R, Lonergan KM, Pusic A, Miller D, Ehlen T, Van Niekerk D, Matisic J, Richards-Kortum R, Follen M et al. Up regulation in gene expression of chromatin remodelling factors in cervical intraepithelial neoplasia. BMC Genomics 2008964. (doi:10.1186/1471-2164-1189-1164)

44 Li C, Ito H, Fujita K, Shiwaku H, Qi Y, Tagawa K, Tamura T \& Okazawa H. Sox2 transcriptionally regulates PQBP1, an intellectual disability-microcephaly causative gene, in neural stem progenitor cells. PLOS ONE 20138 e68627. (doi:10.1371/journal. pone.0068627)

45 Jing X, Infante J, Nachtman RG \& Jurecic R. E3 ligase FLRF (Rnf41) regulates differentiation of hematopoietic progenitors by governing steady-state levels of cytokine and retinoic acid receptors. Experimental Hematology 200836 1110-1120. (doi:10.1016/j. exphem.2008.04.001)

46 Lee HS, Park YY, Cho MY, Chae S, Yoo YS, Kwon MH, Lee CW $\&$ Cho H. The chromatin remodeller RSF1 is essential for PLK1 deposition and function at mitotic kinetochores. Nature Communications 20156 7904. (doi:10.1038/ncomms8904)

47 Jordan NV, Prat A, Abell AN, Zawistowski JS, Sciaky N, Karginova OA, Zhou B, Golitz BT, Perou CM \& Johnson GL. SWI/SNF chromatinremodeling factor Smarcd3/Baf60c controls epithelial-mesenchymal transition by inducing Wnt5a signaling. Molecular and Cellular Biology 201333 3011-3025. (doi:10.1128/MCB.01443-12)

48 Sun X, Xing C, Fu X, Li J, Zhang B, Frierson HF Jr \& Dong JT. Additive effect of Zfhx3/Atbf1 and pten deletion on mouse prostatic tumorigenesis. Journal of Genetics and Genomics 201542 373-382. (doi:10.1016/j.jgg.2015.06.004)

49 Mizutani G, Nakanishi Y, Watanabe N, Honma T, Obana Y, Seki T, Ohni S \& Nemoto N. Expression of somatostatin receptor (SSTR) subtypes (SSTR-1, 2A, 3, 4 and 5) in neuroendocrine tumors using real-time RT-PCR method and immunohistochemistry. Acta Histochemica et Cytochemica 201245 167-176. (doi:10.1267/ ahc.12006)

50 Saveanu A, Muresan M, De Micco C, Taieb D, Germanetti AL, Sebag F, Henry JF, Brunaud L, Enjalbert A, Weryha G et al. Expression of somatostatin receptors, dopamine $\mathrm{D}(2)$ receptors, noradrenaline transporters, and vesicular monoamine transporters in 52 pheochromocytomas and paragangliomas. Endocrine-Related Cancer 201118 287-300. (doi:10.1530/ERC-10-0175)

51 Stridsberg M, Eriksson B, Oberg K \& Janson ET. A comparison between three commercial kits for chromogranin A measurements. Journal of Endocrinology 2003177 337-341. (doi:10.1677/joe.0.1770337)

52 Stridsberg M, Oberg K, Li Q, Engstrom U \& Lundqvist G. Measurements of chromogranin A, chromogranin B (secretogranin I), chromogranin C (secretogranin II) and pancreastatin in plasma and urine from patients with carcinoid tumours and endocrine pancreatic tumours. Journal of Endocrinology 1995144 49-59. (doi:10.1677/ joe.0.1440049)

53 Niec D \& Kunicki PK. Validation of an assay for quantification of free normetanephrine, metanephrine and methoxytyramine in plasma by high performance liquid chromatography with coulometric detection: Comparison of peak-area vs. peak-height measurements. Journal of Chromatography B: Analytical Technologies in the Biomedical and Life Sciences 20151002 63-70. (doi:10.1016/j. jchromb.2015.07.004) 
54 Clarke MR, Weyant RJ, Watson CG \& Carty SE. Prognostic markers in pheochromocytoma. Human Pathology 199829 522-526. (doi:10.1016/S0046-8177(98)90070-3)

55 Angelousi A, Kassi E, Zografos G \& Kaltsas G. Metastatic pheochromocytoma and paraganglioma. European Journal of Clinical Investigation 201545 986-997. (doi:10.1111/eci.12495)

56 Bjorklund P, Pacak K \& Crona J. Precision medicine in pheochromocytoma and paraganglioma: current and future concepts. Journal of Internal Medicine 201610 12507. (doi:10.1111/joim.12507)

57 Kimura N. A pathologist's view: molecular profiles for diagnosing pheochromocytomas and paragangliomas. International Journal of Endocrine Oncology 20152 193-200. (doi:10.2217/ije.15.16)

58 Gilbert JA, Adhikari LJ, Lloyd RV, Rubin J, Haluska P, Carboni JM, Gottardis MM \& Ames MM. Molecular markers for novel therapies in neuroendocrine (carcinoid) tumors. Endocrine-Related Cancer 201017 623-636. (doi:10.1677/ERC-09-0318)

59 Jiao Y, Shi C, Edil BH, de Wilde RF, Klimstra DS, Maitra A, Schulick RD, Tang LH, Wolfgang CL, Choti MA et al. DAXX/ATRX, MEN1, and mTOR pathway genes are frequently altered in pancreatic neuroendocrine tumors. Science 2011331 1199-1203. (doi:10.1126/science.1200609)

60 McDermott JE, Wang J, Mitchell H, Webb-Robertson BJ, Hafen R, Ramey J $\&$ Rodland KD. Challenges in biomarker discovery: combining expert insights with statistical analysis of complex omics data. Expert Opinion on Medical Diagnostics 20137 37-51. (doi:10.1517/17530059.2012.718329)

61 Perren A, Barghorn A, Schmid S, Saremaslani P, Roth J, Heitz PU $\&$ Komminoth P. Absence of somatic SDHD mutations in sporadic neuroendocrine tumors and detection of two germline variants in paraganglioma patients. Oncogene 200221 7605-7608. (doi:10.1038/ sj.onc.1205812)

62 Strong VE, Kennedy T, Al-Ahmadie H, Tang L, Coleman J, Fong Y, Brennan M \& Ghossein RA. Prognostic indicators of malignancy in adrenal pheochromocytomas: clinical, histopathologic, and cell cycle/apoptosis gene expression analysis. Surgery 2008143 759-768. (doi:10.1016/j.surg.2008.02.007)

63 Riester A, Weismann D, Quinkler M, Lichtenauer UD, Sommerey S, Halbritter R, Penning R, Spitzweg C, Schopohl J, Beuschlein F et al. Life-threatening events in patients with pheochromocytoma. European Journal of Endocrinology 2015173 757-764. (doi:10.1530/EJE15-0483)

64 Turkova H, Prodanov T, Maly M, Martucci V, Adams K, Widimsky J Jr, Chen CC, Ling A, Kebebew E, Stratakis CA et al. Characteristics and outcomes of metastatic sdhb and sporadic pheochromocytoma/ paraganglioma: an national institutes of health study. Endocrine Practice 201622 302-314. (doi:10.4158/EP15725.OR)

65 Crona J, Delgado Verdugo A, Maharjan R, Stalberg P, Granberg D, Hellman P \& Bjorklund P. Somatic mutations in H-RAS in sporadic pheochromocytoma and paraganglioma identified by exome sequencing. Journal of Clinical Endocrinology and Metabolism 201398 E1266-E1271. (doi:10.1210/jc.2012-4257)

66 Grimm M, Schmitt S, Teriete P, Biegner T, Stenzl A, Hennenlotter J, Muhs HJ, Munz A, Nadtotschi T, Konig K et al. A biomarker based detection and characterization of carcinomas exploiting two fundamental biophysical mechanisms in mammalian cells. BMC Cancer 201313 569. (doi:10.1186/1471-2407-1113-1569)

Received 25 August 2016

Revised version received 18 October 2016

Accepted 8 November 2016 